\title{
Involvement of the endocannabinoid system in reward processing in the human brain
}

\author{
Hendrika H. van Hell • Gerry Jager • Matthijs G. Bossong • Annelies Brouwer • \\ J. Martijn Jansma • Lineke Zuurman • Joop van Gerven • René S. Kahn • \\ Nick F. Ramsey
}

Received: 30 March 2011 / Accepted: 25 July 2011 /Published online: 6 August 2011

(C) The Author(s) 2011. This article is published with open access at Springerlink.com

\begin{abstract}
Rationale Disturbed reward processing in humans has been associated with a number of disorders, such as depression, addiction, and attention-deficit hyperactivity disorder. The endocannabinoid (eCB) system has been implicated in reward processing in animals, but in humans, the relation between $\mathrm{eCB}$ functioning and reward is less clear.

Objectives The current study uses functional magnetic resonance imaging (fMRI) to investigate the role of the $\mathrm{eCB}$ system in reward processing in humans by examining the effect of the eCB agonist $\Delta^{9}$-tetrahydrocannabinol (THC) on reward-related brain activity.

Methods Eleven healthy males participated in a randomized placebo-controlled pharmacological fMRI study with ad-
\end{abstract}

Electronic supplementary material The online version of this article (doi:10.1007/s00213-011-2428-8) contains supplementary material, which is available to authorized users.

H. H. van Hell · G. Jager · M. G. Bossong • A. Brouwer •

J. M. Jansma $\cdot$ N. F. Ramsey $(\bowtie)$

Department of Neurology and Neurosurgery, G.03.124,

Rudolf Magnus Institute of Neuroscience,

University Medical Center Utrecht,

Heidelberglaan 100,

3584 CX Utrecht, The Netherlands

e-mail: N.Ramsey@umcutrecht.nl

G. Jager

Division of Human Nutrition, Wageningen University,

Wageningen, The Netherlands

L. Zuurman · J. van Gerven

Centre for Human Drug Research,

Leiden, The Netherlands

\section{R. S. Kahn}

Rudolf Magnus Institute of Neuroscience,

Department of Psychiatry, University Medical Center Utrecht,

Utrecht, The Netherlands ministration of THC to challenge the eCB system. We compared anticipatory and feedback-related brain activity after placebo and THC, using a monetary incentive delay task. In this task, subjects are notified before each trial whether a correct response is rewarded ("reward trial") or not ("neutral trial").

Results Subjects showed faster reaction times during reward trials compared to neutral trials, and this effect was not altered by THC. THC induced a widespread attenuation of the brain response to feedback in reward trials but not in neutral trials. Anticipatory brain activity was not affected.

Conclusions These results suggest a role for the eCB system in the appreciation of rewards. The involvement of the eCB system in feedback processing may be relevant for disorders in which appreciation of natural rewards may be affected such as addiction.

Keywords Reward · THC · Endocannabinoid system · fMRI $\cdot$ Monetary reward $\cdot$ Addiction

\section{Introduction}

Disturbed reward processing is associated with a number of psychiatric disorders in humans, such as depression (Domschke et al. 2008), addiction (Pacher et al. 2006), and attention-deficit hyperactivity disorder (Strohle et al. 2008). Animal studies have indicated that the endocannabinoid (eCB) system in the brain plays an important role in reward processing (Solinas et al. 2007). This system consists of $\mathrm{eCB}$ receptors and $\mathrm{eCB}$ ligands that work on these receptors and has a retrograde synaptic effect on the release of various neurotransmitters, such as GABA, glutamate, and dopamine (Pertwee 2008). High densities 
of eCB receptors are found in brain structures associated with reward processing, including the ventral tegmental area, the nucleus accumbens, and prefrontal cortex (Ameri 1999; Gardner 2005). Endocannabinoid agonists have rewarding effects, as has been shown in animals (Gardner 2005; Solinas et al. 2007). Also, blocking the eCB system with the antagonist rimonabant has been shown to reduce the rewarding effects of drugs of abuse such as opiates, nicotine, alcohol, and cocaine, indicating that the eCB system is involved in the neurobiological mechanism underlying drug addiction (De Vries and Schoffelmeer 2005; Maldonado et al. 2006). Further, the cannabinoid agonist $\Delta^{9}$-tetrahydrocannabinol (THC) has rewarding properties by increasing dopamine transmission in the nucleus accumbens (Gardner 2005). However, whether these findings can be extrapolated to humans is unclear.

The location of $\mathrm{eCB}$ receptors in the human brain suggests that the eCB system is also involved in human reward processing (Glass et al. 1997; Terry et al. 2009). An extensive network of brain regions is involved, including limbic structures (notably striatum) and frontal regions (Bjork and Hommer 2007; Knutson et al. 2001a; Knutson and Cooper 2005; O'Doherty 2004). Chronic cannabis use has been shown to blunten the response of the striatum in anticipation of a reward (Van Hell et al. 2010). Similarly, treatment with the $\mathrm{eCB}$ antagonist rimonabant in healthy volunteers resulted in reduced striatal brain activity during reward processing (Horder et al. 2010). Chronic use of rimonabant has been demonstrated to reduce overweight and smoking and to cause depression, but the potential involvement of the reward system is unclear (Cahill and Ussher 2007). However, use of rimonabant in healthy subjects is thwarted by the withdrawal of this drug from registration, following an increased risk of depression and suicide in obese patients (Le Foll et al. 2009). This essentially precludes further use of this drug for elucidating the role of $\mathrm{eCB}$ in human brain function. An alternative approach, where the eCB system is challenged with THC, can also provide a powerful tool for studying its role in reward processing in humans. THC is the main psychoactive constituent of cannabis and possesses rewarding as well as addictive properties (Pertwee 2008). Human studies have shown both increased dopamine transmission as measured with positron emission tomography (PET; Bossong et al. 2009) and no change in striatal dopamine transmission after THC administration, measured with PET (Stokes et al. 2009) or single photon emission tomography (Barkus et al. 2011).

Here, a pharmacological functional magnetic resonance imaging (fMRI) study is presented that examines the involvement of the eCB system in anticipation to a reward as well as reception of the reward. A monetary incentive delay (MID) task is applied (Knutson et al. 2001b), an established reward paradigm which provides a measure of sensitivity to anticipation of reward as well as sensitivity to notification that the reward has been won (Hommer et al. 2011). Previous studies using this paradigm have indicated that anticipation of a reward activates the ventral striatum, and especially the nucleus accumbens, while the reward itself activates frontal brain areas (Knutson et al. 2001a; Knutson et al. 2001b). It was expected that THC would increase baseline dopamine transmission in the reward system. As a result, it was expected that the fMRI response to a natural reward would be decreased, especially in regions in which $\mathrm{eCB}$ receptors are densely distributed, such as the nucleus accumbens and prefrontal cortex.

\section{Methods}

This study is part of the Pharmacological Imaging of the Cannabinoid System (PhICS) project, a comprehensive research project on the role of the endocannabinoid system in the regulation of cognitive brain function in healthy volunteers and patients with psychiatric disorders. Methods of the entire study are reported in detail in a methodological paper (Van Hell et al. 2011). The study is registered in both the EudraCT database (2007-004247-30) and the Dutch Trial Register (NTR1787).

\section{Subjects}

Fourteen healthy male subjects participated in a randomized placebo-controlled cross-over pharmacological MRI study with THC administration. For ethical reasons, subjects needed to be occasional cannabis users (at least four times a year and at most once a week) who never had negative experiences after cannabis use. Subjects were in good health as assessed by medical history, physical examination, electrocardiogram, and routine laboratory tests. In- and exclusion criteria are described in further detail in Van Hell et al. (2011). All volunteers gave written informed consent before entry into the study and were compensated for their participation. The study was approved by the Ethical Committee of the University Medical Centre Utrecht in accordance with the Declaration of Helsinki 2008.

Results are reported on eleven out of the fourteen included subjects. Two data sets were incomplete, due to respectively a technical malfunction of the scanner and feelings of anxiety during the second scanning session. One subject was excluded from analysis due to movement artefacts. Subject characteristics are summarized in Table 1.

\section{Procedure}

At a training session, subjects practiced the procedure of drug administration (inhalation), and participants were 
Table 1 Demographic characteristics and patterns of drug use

\begin{tabular}{lcc}
\hline Subject characteristics $(n=11)$ & Mean \pm SD & Range \\
\hline Age & $21.7 \pm 2.3$ & $18-27$ \\
IQ & $104.5 \pm 6.0$ & $94-111$ \\
Nicotine use last year (cigarettes/week) & $3.0 \pm 8.4$ & $0-28$ \\
Alcohol use last year (units/week) & $16.7 \pm 8.7$ & $2-40$ \\
Cannabis use last year (no. occasions) & $17.9 \pm 13.4$ & $5-52$ \\
Illicit use lifetime (no. occasions) & $1.3 \pm 1.6$ & $0-4$ \\
\hline
\end{tabular}

familiarized with the scan protocol in a mock scanner to reduce stress effects on the following test days. The actual study consisted of two test days, separated by at least 2 weeks to allow for complete clearance of drugs. A standard breakfast or lunch was provided at the beginning of each test day, to ensure equal states of metabolism on both test days. Subjects were instructed not to use cannabis from 2 weeks before the first test day until study completion. Clearance of drugs was tested by means of a urine sample at the beginning of each test day. Additionally, no alcohol was permitted in the $48 \mathrm{~h}$ preceding a test day, and subjects needed to refrain from smoking, eating, and drinking during $4 \mathrm{~h}$ preceding each session.

Drug administration

On test days, subjects received THC or placebo by means of a Volcano ${ }^{\circledR}$ vaporizer (Van Hell et al. 2011; Zuurman et al. 2008) at four time points. The first dose consisted of $6 \mathrm{mg}$ THC or placebo. To maintain average stable levels of intoxicating effects throughout the experiment, upload dosages of $1 \mathrm{mg}$ were used, $30 \mathrm{~min}$ apart, as predicted from previously described dose-effect relationships (Strougo et al. 2008). After the first three administrations of THC or placebo, subjects performed several cognitive tasks during which fMRI scans were obtained. After the last dose of THC or placebo, a battery of neuropsychological tasks was performed (see also Van Hell et al. 2011). Here, results are reported for the monetary incentive delay (MID) task, results of other assessments are reported elsewhere.

\section{Drug effects}

Venous blood samples were collected to determine plasma concentrations of THC and its two most important metabolites, 11-OH-THC and 11-nor-9-carboxy-THC. Blood samples were processed according to Zuurman et al. (2008).

Subjective effects were measured at baseline and before and after each task and throughout the test day using selfreported visual analogue scales (VAS) (Bond and Lader 1974; Bowdle et al. 1998). Heart rate and respiratory function were monitored continuously during scanning. Heart rate was assessed by measuring the electrocardiogram using four electrodes attached to the subject's chest, and respiratory function was assessed by measuring the expansion of a respiration band around the subject's abdomen.

Task

The MID task was based on the paradigm described by Knutson et al. (2001a) (see also Van Hell et al. (2010) and Fig. 1). The task consisted of 48 trials, each lasting $8 \mathrm{~s}$ on average (range 6-12 s). At the beginning of each trial, a cue was presented signaling a trial in which a reward could be won ("reward trial", a circle) or a trial that was never rewarded ("neutral trial", a square). After the cue, a target was presented for a very short time, and subjects had to press a button before the target disappeared. After each reward trial, feedback was given which indicated a successful ("hit") or unsuccessful response ("miss") with the amount of money won (respectively "2 euros" or "0 euro"), as well as the total reward. Anticipation time (the time between cue and target) and inter trial interval were varied $(4.3-10.3 \mathrm{~s}$; mean $6.6 \mathrm{~s}$, and $0-30 \mathrm{~s}$; mean $4.2 \mathrm{~s}$, respectively).

Prior to the experiment, ten practice trials were presented to familiarize subjects with the task. From the practice data, the shortest reaction time to a target was used to determine an individual threshold. Half of the targets in reward trials were presented $200 \mathrm{~ms}$ longer than the individual threshold, and half of the trials $150 \mathrm{~ms}$ shorter to ensure a close to equal number of correct and incorrect responses, to achieve optimal statistical power, as well as a similar total monetary reward for all subjects. Neutral trials were presented with an identical distribution as reward trials.

\section{Scanning parameters}

Image acquisition was performed on a Philips Achieva 3.0 Tesla MR scanner with a Quasar dual gradient set.

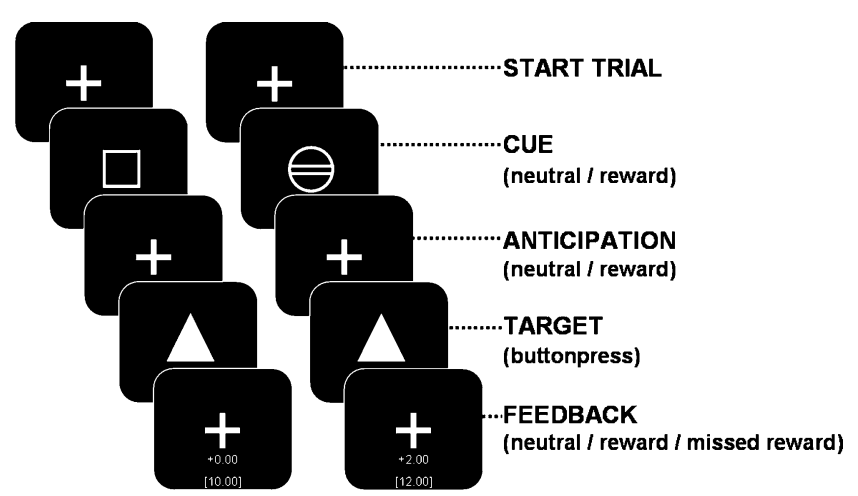

Fig. 1 MID paradigm (see also Van Hell et al. (2010)) 
Functional imaging was performed using a SENSEPRESTO scan protocol (Neggers et al. 2008; scan parameters: TR $22.5 \mathrm{~ms}$; TE $33.2 \mathrm{~ms}$; flip angle $=10^{\circ}$; FOV 224 $\times$ $256 \times 160$; matrix $56 \times 64 \times 40$; voxel size $4.0 \mathrm{~mm}$ isotropic; scan time $0.6075 \mathrm{~s} ; 40$ slices; sagittal orientation, 1,182 volumes). A high-contrast volume with a flip angle of $27^{\circ}$ (FA27) was scanned for registration purposes. Before the functional imaging run, a high-resolution whole brain anatomical scan was performed (scan parameters: TR $9.4 \mathrm{~ms}$; TE $4.7 \mathrm{~ms}$; flip angle $=8^{\circ}$; FOV $220.8 \times 240 \times$ 159.6 ; matrix $368 \times 400 \times 113$; voxel size $0.6 \times 0.6 \times 0.6 \mathrm{~mm}$, 266 slices; sagittal orientation).

\section{Analysis}

\section{Behavioral and physiological measures}

VAS scores were corrected for baseline values and analyzed using repeated-measures ANOVA with drug and time as within-subject factors (Van Hell et al. 2011; Zuurman et al. 2008). Mean heart rate during the MID task was calculated for placebo and THC sessions separately.

\section{Task performance}

Reward task performance was measured using reaction times (RT) on neutral and rewarding trials. A repeatedmeasures analysis with drug (two levels: THC and placebo) and condition (two levels: reward and neutral) was performed to analyze differences between THC and placebo, and rewarding and neutral trials.

\section{fMRI}

Functional MRI data were preprocessed and analyzed using SPM5 (Wellcome Trust Centre for Neuroimaging, London, UK). Preprocessing of data consisted of realignment of functional images and coregistration with the anatomical volume using the FA27 volume. After realignment, functional scans were spatially normalized into standard MNI space and smoothed (FWHM $=8 \mathrm{~mm}$ ) to reduce the effect of between-subject spatial variability in activation.

For each individual subject, regression-coefficients for each voxel were obtained from a general linear model regression analysis using a factor matrix that contained factors representing event-related changes time-locked to anticipation and feedback of neutral and reward trials (hits and misses modelled separately). All factors were convolved with a canonical hemodynamic response function. For anticipation, the variable anticipation period was used as the expected duration of brain activity. For feedback, a fixed period (duration of feedback period) was used as the expected duration of brain activity. To reduce the presence of slow trends in the signal, a high-pass filter with a cut-off frequency of $0.007 \mathrm{~Hz}$ was applied to the data.

As this is the first study exploring the effects of THC administration on reward processing in the brain, we chose to perform region of interest (ROI) analyses on areas that were involved in this particular task. This approach has been described previously as a powerful approach to explore data in a complex design (see Poldrack (2007)). Group activation maps were created for placebo and THC sessions separately. ROIs were calculated based on two contrasts that were sensitive for signal changes related to reward. The first group map contrasted anticipation of rewarding targets versus anticipation of neutral targets (denoted as "ANT"). The second group map contrasted feedback of rewarded targets versus feedback of missed targets (denoted as "FB"). ROIs were constructed by clustering neighbouring voxels that reached threshold in either the placebo or the THC session (ANT thresholded at $t>3.2, p<0.005$; FB thresholded at $t>4.1, p<0.001)$. Constructing the ROIs based on the highest values in either the THC or the placebo session prevents bias towards our hypothesis (Kriegeskorte et al. 2009; Vul et al. 2009). Mean signal change for each ROI, each subject and each condition were based on beta values averaged over voxels in each ROI, extracted using Marsbar (Brett et al. 2002).

All hypothesis tests were performed using SPSS 15 . To measure THC effects on anticipation, an overall repeatedmeasures MANOVA was performed on ANT ROIs with drug (two levels: THC and placebo), condition (two levels: reward and neutral), and ROI (fourteen levels) as withinsubjects factors. Follow-up ANOVA analyses were performed for every ROI separately with drug and condition as within-subjects factors.

To measure effects of THC on reward feedback activity, repeated-measures MANOVA were performed on FB ROIs, for neutral and reward trials separately, with drug (two levels), condition (two levels: hits and misses), and ROI (ten levels) as within-subjects factors. Follow-up ANOVA analyses were again performed for every ROI.

\section{Results}

Behavioral results

THC plasma concentration reached a maximum of $60.1 \pm$ $33.7 \mathrm{ng} / \mathrm{ml} 5 \mathrm{~min}$ after inhalation of $6 \mathrm{mg}$ THC and decreased rapidly thereafter (also see Van Hell et al. (2011)).

Repeated-measures ANOVA with drug (two levels) as within-subject factor showed that THC administration increased subjective scores of "feeling high" $(F(1,10)=$ $10.4, p<0.01)$ and heart rate $(F(1,10)=8.0, p<0.02)$. THC 
Table 2 Physiological and behavioral effects of placebo and THC $($ mean \pm SD)

\begin{tabular}{lrcl}
\hline & Placebo & \multicolumn{1}{l}{ THC } & $p$ \\
\hline Heart rate (beats per minute) & $68.9 \pm 8.5$ & $83.8 \pm 20.8$ & $0.018^{\mathrm{a}}$ \\
VAS feeling high & $0.2 \pm 0.8$ & $19.5 \pm 19.9$ & $0.009^{\mathrm{a}}$ \\
VAS internal perception & $-0.4 \pm 1.5$ & $1.3 \pm 2.7$ & 0.086 \\
VAS external perception & $1.5 \pm 2.5$ & $6.7 \pm 9.6$ & 0.13 \\
VAS alertness & $-1.5 \pm 7.8$ & $-10.6 \pm 9.7$ & $0.028^{\mathrm{a}}$ \\
VAS contentedness & $-1.5 \pm 4.7$ & $-4.2 \pm 4.8$ & 0.11 \\
VAS calmness & $1.5 \pm 7.9$ & $1.8 \pm 10.7$ & 0.94 \\
\hline
\end{tabular}

${ }^{\text {a }}$ Significant difference between THC and placebo

decreased "alertness" $(F(1,10)=6.6, p<0.03)$ and induced a trend towards increased "internal perception" (reflecting inner feelings that do not correspond with reality) $(F(1,10)=3.6, p<0.09)$ (see Table 2).

Performance MID task

Repeated-measures ANOVA with drug (two levels) and condition (two levels) as within-subject factors showed a significant effect of condition $(F(1,10)=22.0, p=$ 0.001 ), indicating that subjects were faster on reward trials than on neutral trials, and a near significant effect of drug $(F(1,10)=4.5, p=0.06)$, indicating that subjects were slower after THC administration compared to placebo (see Fig. 2). A post hoc paired $t$ test per condition indicated that this effect was most pronounced during reward trials $(t=2.2, p=0.051)$.

\section{fMRI results}

\section{Anticipation}

ANT ROIs included left and right caudate nucleus, left and right parietal cortex, middle cingulate, left and right cerebellum, left pre/postcentral gyrus, left insula, anterior cingulate/supplementary motor area, right inferior orbitofrontal gyrus, right middle and inferior frontal gyrus, and the thalamus/brain stem (see Fig. 3a and Figure S1 and Table S1a).

Repeated-measures MANOVA revealed no significant effect of drug $(F=0.01, p=0.9)$ or drug by condition $(F=$ $0.03, p=0.9$ ) (see Fig. 4a). A significant drug by condition by ROI interaction effect $(F=2.5, p<0.05)$ indicated that drug by condition effects differed between ROIs. Follow-up analysis per ROI (not corrected for multiple comparisons) revealed no significant drug effect in individual ROIs. One ROI, the right inferior orbitofrontal gyrus, showed a significant drug by condition interaction effect $(F=7.9, p<$ 0.05 ; see Fig. $4 \mathrm{~b}$ and Table $\mathrm{S} 1 \mathrm{~b}$ ). This interaction was a result of a larger signal increase in anticipation of a reward after THC administration than after placebo administration. However, the individual ROI effect did not survive correction for multiple comparisons.

\section{Feedback}

FB ROIs included the inferior parietal and temporal gyrus bilaterally, posterior and anterior cingulate, middle orbitofrontal gyrus, and right superior frontal gyrus (see Fig. 3b and Figure S2 and Table S2a). No differences were found in FB ROIs between THC and placebo during neutral feedback (see Fig. 5a). A repeated-measures MANOVA showed that during reward trials, THC administration caused a significant reduction in reward-related brain activity (drug effect, $F=13.1 ; p<0.01$; see Fig. 5a), indicating that THC reduced the signal for hits as well as misses. Further analysis per ROI (not corrected for multiple comparisons; see Fig. 5b and Table S2b) showed that this main drug effect was present in the left inferior parietal cortex $(F=5.5, p<0.05)$, inferior temporal gyrus bilaterally (left, $F=8.2, p<0.05$ and right, $F=7.4, p<0.05$ ), and at trend level in the posterior cingulate $(F=4.8, p<0.1)$ and right inferior parietal cortex $(F=4.2, p<0.1)$.

In addition, an overall interaction effect of drug by condition by ROI $(F=4.6 ; p=0.001)$ was found, indicating that the drug by condition effect differed between ROIs. This interaction was a result of the fact that two different drug by condition interaction effects were found in individual ROIs: THC decreased the signal

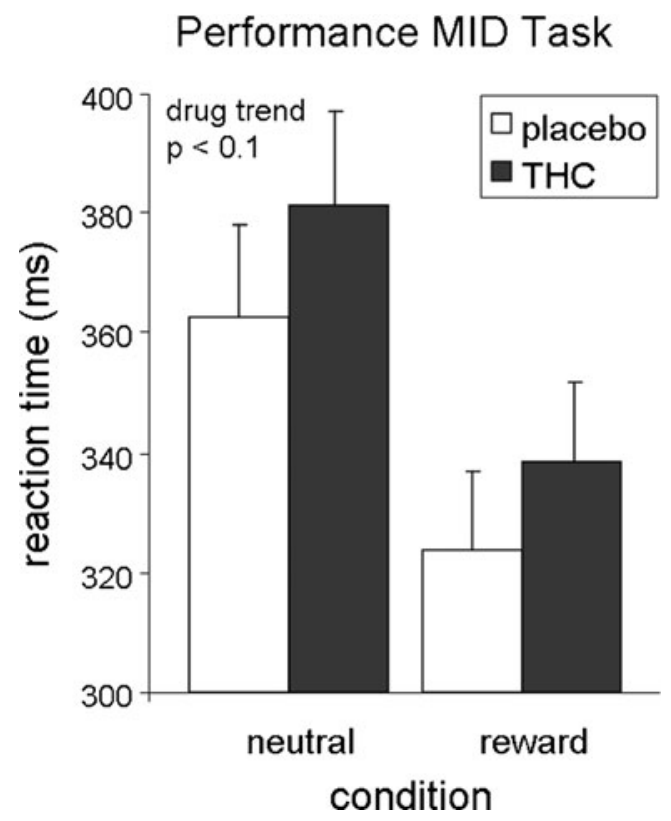

Fig. 2 Performance of the MID task. Error bars denote Standard Error of the Mean (SEM) 
Fig. 3 Regions of interest; based on pooled group activation maps of THC and placebo; a reward anticipation; $\mathbf{b}$ reward feedback. $L=$ left, $R=$ right
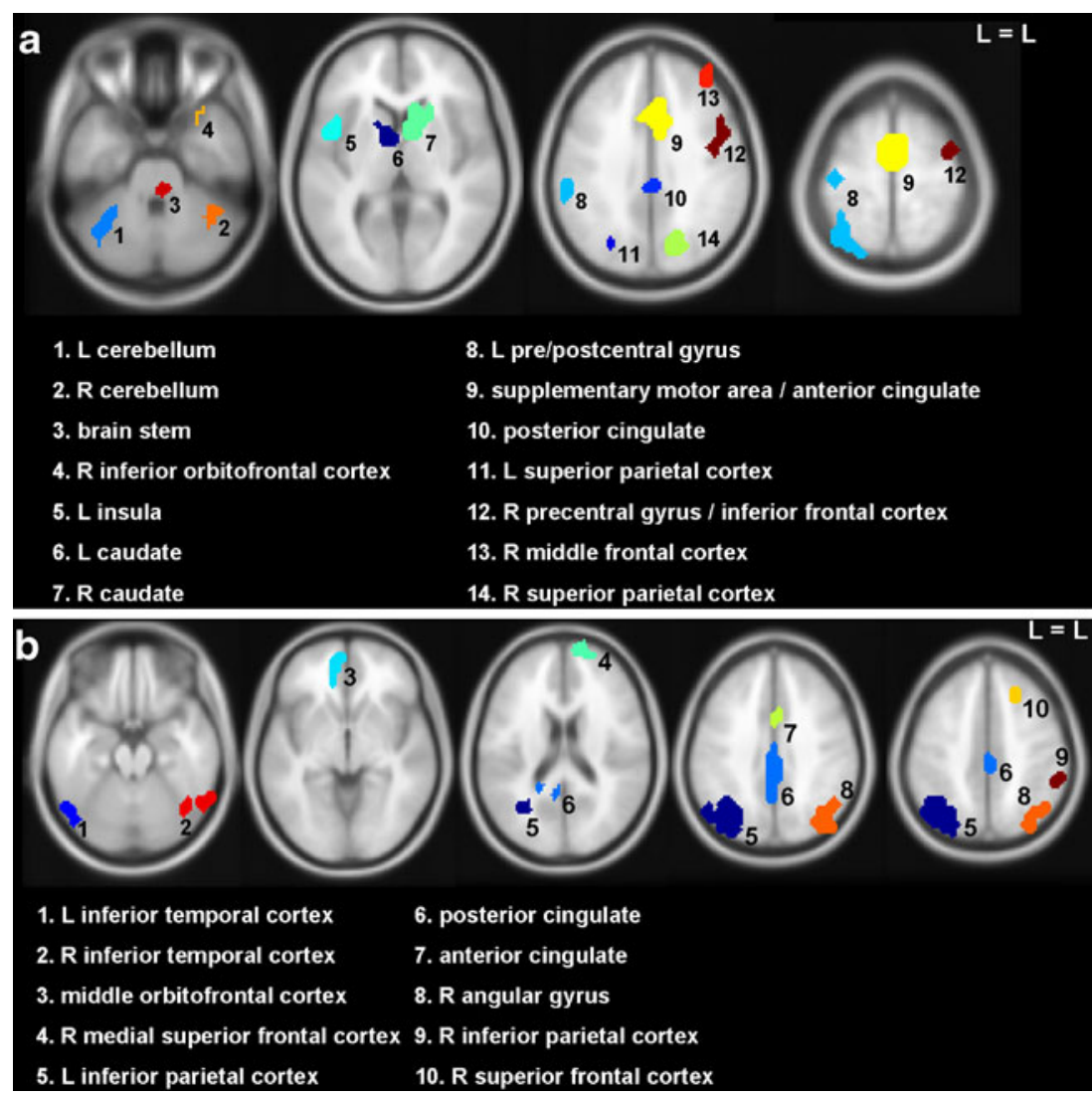

change for a miss but not for a hit in the posterior cingulate $(F=6.3, p<0.05)$ and the middle orbitofrontal

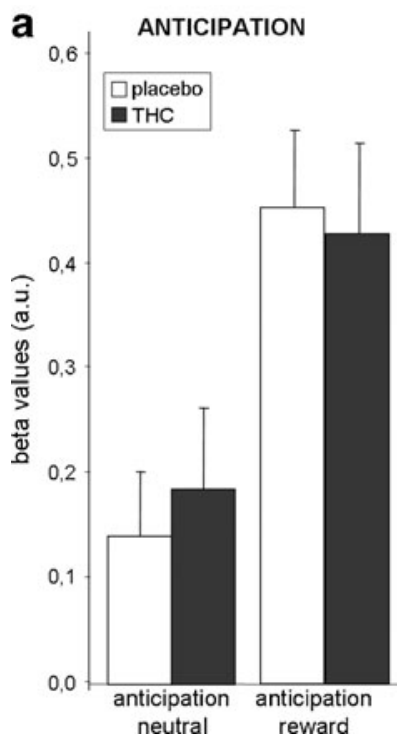

b

R Inf Orbitofrontal Cortex

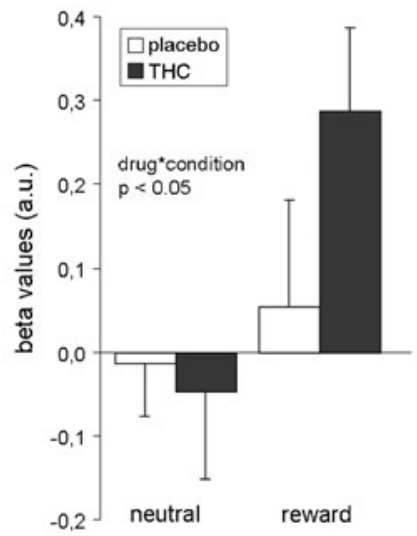

Fig. 4 Brain activity during anticipation. a Brain activity during anticipation averaged across ROIs; b right inferior orbitofrontal cortex. Error bars denote standard error of the mean (SEM). a.u.= arbitrary units; $R=$ right; $I n f=$ inferior. $p$ values are not corrected for multiple comparisons cortex $(F=12.6, p<0.01)$, while THC reduced the signal change related to a hit but not a miss in the right superior frontal cortex $(F=6.7, p<0.05)$. A trend for an interaction effect of drug by condition in the left inferior temporal gyrus indicated a larger attenuation of brain activity during hits than misses after THC administration $(F=3.5 ; p<0.1)$. However, none of these ROI effects survived correction for multiple comparisons (see Tables 3, 4, S1b and S2b).

\section{Discussion}

In this study, the role of the $\mathrm{eCB}$ system in reward processing in humans was examined by assessing the effects of THC administration on brain activity during monetary reward anticipation and feedback. Subjects showed similar behavioral responses to reward during THC and placebo, with faster responses if a reward could be won. THC administration attenuated brain activity during reward feedback compared to placebo. THC did not affect brain activity related to feedback if there was no possibility to win a reward. These results indicate that THC administration predominantly reduces the effect of feedback in situations where there is the possibility to earn a reward and suggest involvement of the eCB system in appreciation of a received reward. This effect was largest in the inferior 


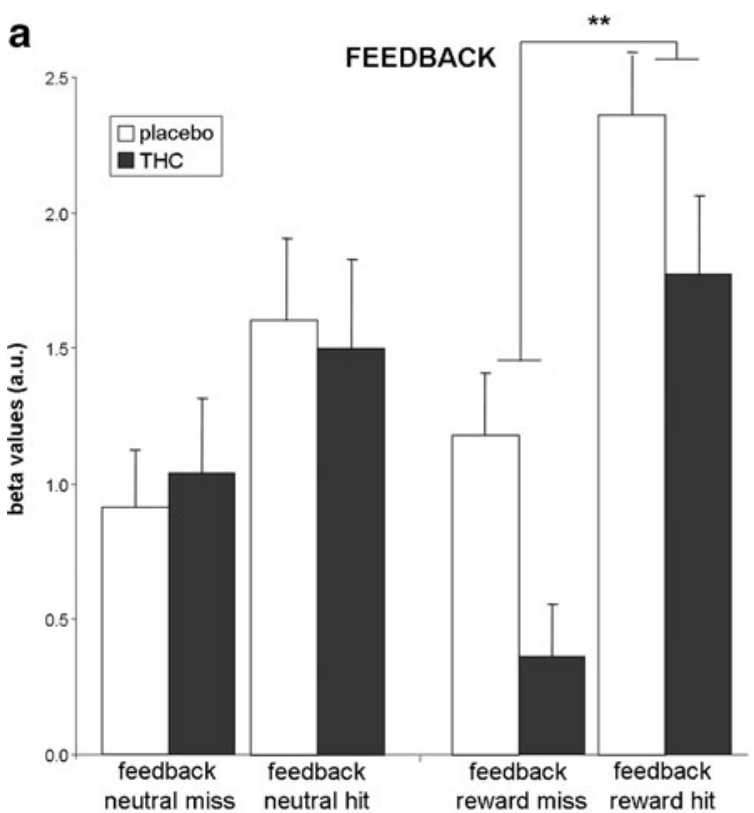

Fig. 5 Brain activity during feedback. a Brain activity during feedback averaged across ROIs; $\mathbf{b}$ reward feedback in separate ROIs. Error bars denote standard error of the mean (SEM). $L=$ left, $R=$ right; Inf $=$ inferior; Sup $=$ superior; Post=posterior; $C x=$ cortex; $a . u$. $=$ arbi-

parietal and temporal cortex. The inferior parietal cortex is a pivotal part of the attention network (Naghavi and Nyberg 2005; Pessoa et al. 2002). More specifically, the inferior parietal cortex is associated with a salience representation of the outside world (Gottlieb 2007), indicating that attention is directed towards salient- or task-relevant objects. Previous studies have shown that eCB affects inferior parietal cortex function, as decreased activity has been reported after THC administration in association with
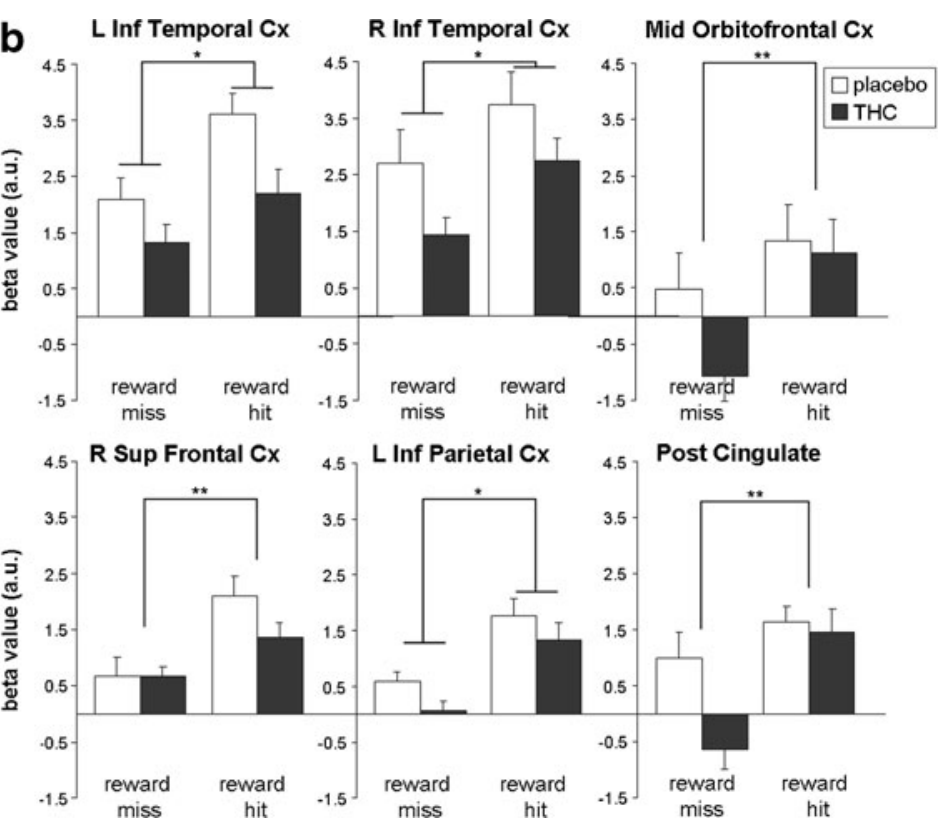

trary units. Single asterisk significant drug effect $(p<0.05)$; double asterisk significant drug by condition interaction effect $(p<0.05) . p$ values are not corrected for multiple comparisons

auditory attention (Roser et al. 2008) and during emotional processing (Fusar-Poli et al. 2009). Hence, our result suggests a general effect of THC on attentional processes during salient feedback.

Subjects reacted faster on the task when a reward could be won, during both THC and placebo, an effect that has been reported previously (Knutson et al. 2001b; Bjork and Hommer 2007). This indicates that subjects were motivated to perform the task during both placebo and THC. However,
Table 3 Regions of interest during reward anticipation

Max $T$ maximum $T$ value, $R$ right, $L$ left

\begin{tabular}{|c|c|c|c|c|c|c|}
\hline & \multirow[t]{2}{*}{ Area } & \multirow[t]{2}{*}{$\operatorname{Max} T$} & \multirow[t]{2}{*}{ Cluster size } & \multicolumn{3}{|c|}{ MNI coordinates } \\
\hline & & & & $x$ & $y$ & $z$ \\
\hline 1 & L cerebellum & 4.6 & 42 & -32 & -52 & -32 \\
\hline 2 & $\mathrm{R}$ cerebellum & 4.2 & 31 & 44 & -48 & -24 \\
\hline 3 & Brain stem & 4.5 & 68 & 0 & -20 & -12 \\
\hline 4 & $\mathrm{R}$ inferior orbitofrontal cortex & 8.7 & 27 & 28 & 24 & -24 \\
\hline 5 & $\mathrm{~L}$ insula & 6.5 & 49 & -44 & 12 & 8 \\
\hline 6 & L caudate & 4.2 & 30 & -8 & 4 & 4 \\
\hline 7 & $\mathrm{R}$ caudate & 6.0 & 70 & 12 & 8 & 0 \\
\hline 8 & L pre/postcentral gyrus & 4.4 & 209 & -48 & -24 & 56 \\
\hline 9 & SMA/anterior cingulate & 5.8 & 326 & 0 & -4 & 60 \\
\hline 10 & Posterior cingulate & 4.8 & 36 & -12 & -72 & 44 \\
\hline 11 & L superior parietal cortex & 5.5 & 65 & -40 & -60 & 60 \\
\hline 12 & $\mathrm{R}$ precentral gyrus & 7.1 & 123 & 48 & 0 & 40 \\
\hline 13 & $\mathrm{R}$ middle frontal cortex & 7.9 & 37 & 40 & 44 & 32 \\
\hline 14 & $\mathrm{R}$ superior parietal cortex & 4.9 & 71 & 12 & -72 & 44 \\
\hline
\end{tabular}


Table 4 Regions of interest during reward feedback

\begin{tabular}{|c|c|c|c|c|c|c|}
\hline & \multirow[t]{2}{*}{ Area } & \multirow[t]{2}{*}{$\operatorname{Max} T$} & \multirow[t]{2}{*}{ Cluster size } & \multicolumn{3}{|c|}{ MNI coordinates } \\
\hline & & & & $x$ & $y$ & $z$ \\
\hline 1 & $\mathrm{~L}$ inferior temporal cortex & 8.5 & 43 & -56 & -64 & -20 \\
\hline 2 & $\mathrm{R}$ inferior temporal cortex & 8.3 & 51 & 60 & -60 & -20 \\
\hline 3 & Middle orbitofrontal cortex & 6.1 & 31 & -4 & 60 & -4 \\
\hline 4 & Medial superior frontal cortex & 8.7 & 30 & 16 & 68 & 16 \\
\hline 5 & $\mathrm{~L}$ inferior parietal cortex & 15.4 & 350 & -32 & -68 & 36 \\
\hline 6 & Posterior cingulate & 6.4 & 202 & -4 & -48 & 28 \\
\hline 7 & Anterior cingulate & 6.1 & 30 & 0 & 8 & 32 \\
\hline 8 & $\mathrm{R}$ angular gyrus & 8.3 & 132 & 40 & -68 & 40 \\
\hline 9 & $\mathrm{R}$ inferior parietal cortex & 8.7 & 39 & 56 & -40 & 48 \\
\hline 10 & $\mathrm{R}$ superior frontal cortex & 5.9 & 27 & 16 & 20 & 52 \\
\hline
\end{tabular}

subjects were slower after THC administration during both neutral and reward trials, indicating that $\mathrm{THC}$ has a more generalized motor or attentional effect in addition to the reward-specific effects observed in the present study. Indeed, another study assessing the effects of acute THC administration reported that $\mathrm{THC}$ can increase reaction times on a number of tasks assessing either memory, attention, or simple reaction times (Curran et al. 2002).

Analyses of individual ROIs yielded some effects of THC (described in the "Results" section), but none survived the corrected threshold for multiple comparisons. They do suggest that brain activity during feedback was attenuated most in the posterior cingulate, the superior frontal cortex, and orbitofrontal cortex. These have all been associated with reward processing (Hayden et al. 2008; McCoy and Platt 2005; Tabuchi et al. 2005; Dom et al. 2005; Egerton et al. 2005; Wallis and Kennerley 2010) or effects of THC administration (Fusar-Poli et al. 2009; Stokes et al. 2010). Nevertheless, the most robust finding was a network-wide effect of THC.

The eCB system has been implicated in various aspects of addiction, such as drug-seeking and relapse (De Vries and Schoffelmeer 2005). Animal studies showed that activating the eCB system with an agonist provoked relapse to use of other drugs, suggesting a generic but complex role for eCB in reward (Wiskerke et al. 2008). Blocking the eCB system with the antagonist rimonabant led to the opposite effect, reducing drug-seeking and relapse (De Vries et al. 2001). One could argue that if THC activates the eCB system and thereby induces mild elevation of activity of the reward system (Bossong et al. 2009; Stokes et al. 2010), the impact of other rewarding stimuli may be dampened as a result, thus explaining the attenuation of brain activity due to a monetary reward. An indication for such a mechanism can be derived from the effect of chronic cannabis use on motivation in general. Although there is no clear evidence for loss of motivation, the negative effects of chronic cannabis use on school performance and later life outcomes may be seen as an indication (Fergusson and Boden 2008), but this interpretation warrants further investigation.

In our study, anticipation of a reward activated the striatum, insula, anterior cingulate, and frontal brain regions, and feedback of reward activated the posterior and anterior cingulate, inferior parietal cortex, orbitofrontal, and superior frontal cortex. This pattern of brain activity is in line with previous reward imaging studies, which have reported striatal activity during anticipation and frontal activity during feedback of reward (Bjork and Hommer 2007; Knutson et al. 2001a; Knutson and Cooper 2005; O'Doherty 2004).

The nucleus accumbens did not show significantly elevated activity during reward anticipation in our study. Additional ROI analyses with the nucleus accumbens as an anatomically defined ROI also did not show a significant effect of THC (data not shown). Factors that may have reduced effects of reward in the striatum and nucleus accumbens include the fact that subjects were paid 250 euros for participation in the study. An extra reward of 24 euros that was won during the reward task may have been too small in comparison. Specifically for the nucleus accumbens, it can be noted that fMRI measurements in this region tend to be less reliable, as it is located close to the nasal cavity, which reduces the BOLD signal to noise ratio.

The significant anticipatory effect of reward in striatum was slightly reduced after $\mathrm{THC}$, but this effect was too small to become significant. This is contrary to what we expected, as the striatum shows high densities of CB1 receptors, and $\mathrm{THC}$ is known to elicit rewarding and dopamine-elevating effects on the striatum (Bossong et al. 2009; Gardner 2005). However, recent studies in humans indicated that the role of THC in increasing dopamine levels in the striatum may be limited (Stokes et al. 2009; Barkus et al. 2011). Effects of THC in the striatum may also have been influenced by the fact that our subjects were 
occasional users of cannabis (see also Van Hell et al. 2010). It may also be possible that the effect of THC on striatal activity is domain specific, and limited for anticipatory reward activity. There have not been any previous studies examining effects of THC on striatal reward processing activity to compare our results to, but previous studies using different cognitive paradigms have shown attenuation of ventrostriatal activity after THC during retrieval of memory (Bhattacharyya et al. 2009) and an increase in brain activity in the caudate during response inhibition (Borgwardt et al. 2008), which would be in line with the hypothesis that THC effects may be domain specific.

The results from the current study should be interpreted with due care. Our sample size was small for an fMRI study which compares data between sessions. A larger sample could have revealed more effects of THC, for instance, during task anticipation in striatal areas. In addition, although the study was designed to be double blind, THC induced behavioral effects that were identified by most subjects, possibly causing expectancy effects across sessions. The influence of expectancy was minimized by using a randomized cross-over design, thus balancing expectancy effects across sessions. Still, it cannot be excluded that expectancy effects may have affected our results to some extent.

In conclusion, this study provides new arguments for eCB involvement in the reward system in humans. Findings suggest that $\mathrm{THC}$ affects appreciation of obtaining a monetary reward. The involvement of the eCB system in feedback processing may be relevant for disorders in which appreciation of natural rewards may be affected such as addiction.

Acknowledgements The PhICS study is performed within the framework of Top Institute Pharma, project number T5-107. We thank Storz and Bickel for kindly supplying the Volcano vaporizer and Emi Saliasi, David Terburg, and Linda Klumpers for help with data acquisition and analysis.

Conflicts of interest Authors report no conflicts of interest.

Open Access This article is distributed under the terms of the Creative Commons Attribution Noncommercial License which permits any noncommercial use, distribution, and reproduction in any medium, provided the original author(s) and source are credited.

\section{References}

Ameri A (1999) The effects of cannabinoids on the brain. Prog Neurobiol 58:315-348

Barkus E, Morrison PD, Vuletic D, Dickson J, Ell PJ, Pilowsky LS, Brenneisen R, Holt DW, Powell J, Kapur S, Murray RM (2011) Does intravenous $\Delta$ 9-tetrahydrocannabinol increase dopamine release? A SPET study. J.Psychopharmacol. doi:10.1177/ 0269881110382465 (in press)

Bhattacharyya S, Fusar-Poli P, Borgwardt S, Martin-Santos R, Nosarti C, O'Carroll C, Allen P, Seal ML, Fletcher PC, Crippa JA,
Giampietro V, Mechelli A, Atakan Z, McGuire P (2009) Modulation of mediotemporal and ventrostriatal function in humans by Delta9-tetrahydrocannabinol: a neural basis for the effects of Cannabis sativa on learning and psychosis. Arch Gen Psychiatry 66:442-451

Bjork JM, Hommer DW (2007) Anticipating instrumentally obtained and passively-received rewards: a factorial fMRI investigation. Behav Brain Res 177:165-170

Bond A, Lader M (1974) The use of analogue scales in rating subjective feelings. Br J Med Psychol 47:211-218

Borgwardt SJ, Allen P, Bhattacharyya S, Fusar-Poli P, Crippa JA, Seal ML, Fraccaro V, Atakan Z, Martin-Santos R, O'Carroll C, Rubia K, McGuire PK (2008) Neural basis of Delta-9-tetrahydrocannabinol and cannabidiol: effects during response inhibition. Biol Psychiatry 64:966-973

Bossong MG, van Berckel BN, Boellaard R, Zuurman L, Schuit RC, Windhorst AD, van Gerven JM, Ramsey NF, Lammertsma AA, Kahn RS (2009) Delta 9-tetrahydrocannabinol induces dopamine release in the human striatum. Neuropsychopharmacology 34:759-766

Bowdle TA, Radant AD, Cowley DS, Kharasch ED, Strassman RJ, Roy-Byrne PP (1998) Psychedelic effects of ketamine in healthy volunteers: relationship to steady-state plasma concentrations. Anesthesiology 88:82-88

Brett M, Anton J-L, Valabregue R, Poline J-B (2002) Region of interest analysis using an SPM toolbox. [abstract] Presented at the 8th International Conference on Functional Mapping of the Human Brain. NeuroImage 16(2)

Cahill K, Ussher M (2007) Cannabinoid type 1 receptor antagonists (rimonabant) for smoking cessation. Cochrane Database Syst Rev CD005353

Curran HV, Brignell C, Fletcher S, Middleton P, Henry J (2002) Cognitive and subjective dose-response effects of acute oral Delta 9-tetrahydrocannabinol (THC) in infrequent cannabis users. Psychopharmacology (Berl) 164:61-70

De Vries TJ, Schoffelmeer AN (2005) Cannabinoid CB1 receptors control conditioned drug seeking. Trends Pharmacol Sci 26:420-426

De Vries TJ, Shaham Y, Homberg JR, Crombag H, Schuurman K, Dieben J, Vanderschuren LJ, Schoffelmeer AN (2001) A cannabinoid mechanism in relapse to cocaine seeking. Nat Med 7:1151-1154

Dom G, Sabbe B, Hulstijn W, van den Brink W (2005) Substance use disorders and the orbitofrontal cortex: systematic review of behavioural decision-making and neuroimaging studies. $\mathrm{Br} \mathrm{J}$ Psychiatry 187:209-220

Domschke K, Dannlowski U, Ohrmann P, Lawford B, Bauer J, Kugel H, Heindel W, Young R, Morris P, Arolt V, Deckert J, Suslow T, Baune BT (2008) Cannabinoid receptor 1 (CNR1) gene: impact on antidepressant treatment response and emotion processing in major depression. Eur Neuropsychopharmacol 18:751-759

Egerton A, Brett RR, Pratt JA (2005) Acute delta9-tetrahydrocannabinolinduced deficits in reversal learning: neural correlates of affective inflexibility. Neuropsychopharmacology 30:1895-1905

Fergusson DM, Boden JM (2008) Cannabis use and later life outcomes. Addiction 103:969-976

Fusar-Poli P, Crippa JA, Bhattacharyya S, Borgwardt SJ, Allen P, Martin-Santos R, Seal M, Surguladze SA, O'Carrol C, Atakan Z, Zuardi AW, McGuire PK (2009) Distinct effects of delta-9tetrahydrocannabinol and cannabidiol on neural activation during emotional processing. Arch Gen Psychiatry 66:95-105

Gardner EL (2005) Endocannabinoid signaling system and brain reward: emphasis on dopamine. Pharmacol Biochem Behav 81:263-284

Glass M, Dragunow M, Faull RL (1997) Cannabinoid receptors in the human brain: a detailed anatomical and quantitative autoradiographic study in the fetal, neonatal and adult human brain. Neuroscience 77:299-318

Gottlieb J (2007) From thought to action: the parietal cortex as a bridge between perception, action, and cognition. Neuron 53:9-16 
Hayden BY, Nair AC, McCoy AN, Platt ML (2008) Posterior cingulate cortex mediates outcome-contingent allocation of behavior. Neuron 60:19-25

Hommer DW, Bjork JM, Gilman JM (2011) Imaging brain response to reward in addictive disorders. AnnNYAcadSci 1216:50-61

Horder J, Harmer CJ, Cowen PJ, McCabe C (2010) Reduced neural response to reward following 7 days treatment with the cannabinoid $\mathrm{CB}(1)$ antagonist rimonabant in healthy volunteers. Int J Neuropsychopharmacol 13:1103-1113

Knutson B, Adams CM, Fong GW, Hommer D (2001a) Anticipation of increasing monetary reward selectively recruits nucleus accumbens. JNeurosci 21:RC159

Knutson B, Cooper JC (2005) Functional magnetic resonance imaging of reward prediction. Curr Opin Neurol 18:411-417

Knutson B, Fong GW, Adams CM, Varner JL, Hommer D (2001b) Dissociation of reward anticipation and outcome with eventrelated fMRI. Neuroreport 12:3683-3687

Kriegeskorte N, Simmons WK, Bellgowan PS, Baker CI (2009) Circular analysis in systems neuroscience: the dangers of double dipping. Nat Neurosci 12:535-540

Le Foll B, Gorelick DA, Goldberg SR (2009) The future of endocannabinoid-oriented clinical research after CB1 antagonists. Psychopharmacology (Berl) 205:171-174

Maldonado R, Valverde O, Berrendero F (2006) Involvement of the endocannabinoid system in drug addiction. Trends Neurosci 29:225-232

McCoy AN, Platt ML (2005) Expectations and outcomes: decisionmaking in the primate brain. J Comp Physiol A Neuroethol Sens Neural Behav Physiol 191:201-211

Naghavi HR, Nyberg L (2005) Common fronto-parietal activity in attention, memory, and consciousness: shared demands on integration? Conscious Cogn 14:390-425

Neggers SF, Hermans EJ, Ramsey NF (2008) Enhanced sensitivity with fast three-dimensional blood-oxygen-level-dependent functional MRI: comparison of SENSE-PRESTO and 2D-EPI at 3 T. NMR Biomed 21:663-676

O'Doherty JP (2004) Reward representations and reward-related learning in the human brain: insights from neuroimaging. Curr Opin Neurobiol 14:769-776

Pacher P, Batkai S, Kunos G (2006) The endocannabinoid system as an emerging target of pharmacotherapy. Pharmacol Rev 58:389-462

Pertwee RG (2008) Ligands that target cannabinoid receptors in the brain: from $\mathrm{THC}$ to anandamide and beyond. Addict Biol 13:147-159

Pessoa L, Kastner S, Ungerleider LG (2002) Attentional control of the processing of neural and emotional stimuli. Brain Res Cogn Brain Res 15:31-45

Poldrack RA (2007) Region of interest analysis for fMRI. Soc Cogn Affect Neurosci 2:67-70

Roser P, Juckel G, Rentzsch J, Nadulski T, Gallinat J, Stadelmann AM (2008) Effects of acute oral Delta9-tetrahydrocannabinol and standardized cannabis extract on the auditory P300 event-related potential in healthy volunteers. Eur Neuropsychopharmacol 18:569-577

Solinas M, Yasar S, Goldberg SR (2007) Endocannabinoid system involvement in brain reward processes related to drug abuse. Pharmacol Res 56:393-405

Stokes PRA, Mehta MA, Curran HV, Breen G, Grasby PM (2009) Can recreational doses of THC produce significant dopamine release in the human striatum? Neuroimage 48:186-190

Stokes PR, Egerton A, Watson B, Reid A, Breen G, Lingford-Hughes A, Nutt DJ, Mehta MA (2010) Significant decreases in frontal and temporal $[11 \mathrm{C}]$-raclopride binding after THC challenge. Neuroimage 52:1521-1527

Strohle A, Stoy M, Wrase J, Schwarzer S, Schlagenhauf F, Huss M, Hein J, Nedderhut A, Neumann B, Gregor A, Juckel G, Knutson B, Lehmkuhl U, Bauer M, Heinz A (2008) Reward anticipation and outcomes in adult males with attention-deficit/hyperactivity disorder. Neuroimage 39:966-972

Strougo A, Zuurman L, Roy C, Pinquier JL, van Gerven JM, Cohen AF, Schoemaker RC (2008) Modelling of the concentrationeffect relationship of THC on central nervous system parameters and heart rate-insight into its mechanisms of action and a tool for clinical research and development of cannabinoids. J Psychopharmacol 22:717-726

Tabuchi E, Furusawa AA, Hori E, Umeno K, Ono T, Nishijo H (2005) Neural correlates to action and rewards in the rat posterior cingulate cortex. Neuroreport 16:949-953

Terry GE, Liow JS, Zoghbi SS, Hirvonen J, Farris AG, Lerner A, Tauscher JT, Schaus JM, Phebus L, Felder CC, Morse CL, Hong JS, Pike VW, Halldin C, Innis RB (2009) Quantitation of cannabinoid $\mathrm{CB} 1$ receptors in healthy human brain using positron emission tomography and an inverse agonist radioligand. Neuroimage 48:362-370

Van Hell HH, Bossong MG, Jager G, Kahn RS, Ramsey NF (2011) Methods of the Pharmacological Imaging of the Cannabinoid System (PhICS) study: towards understanding the role of the brain endocannabinoid system in human cognition. International Journal of Methods in Psychiatric Research (in press)

Van Hell HH, Vink M, Ossewaarde L, Jager G, Kahn RS, Ramsey NF (2010) Chronic effects of cannabis use on the human reward system: an fMRI study. Eur Neuropsychopharmacol 20:153-163

Vul E, Harris C, Winkielman P, Pashler H (2009) Puzzlingly high correlations in fMRI studies of emotion, personality, and social cognition. Perspectives on Psychological Science 4:274-290

Wallis JD, Kennerley SW (2010) Heterogeneous reward signals in prefrontal cortex. Curr Opin Neurobiol 20:191-198

Wiskerke J, Pattij T, Schoffelmeer AN, De Vries TJ (2008) The role of CB1 receptors in psychostimulant addiction. Addict Biol 13:225-238

Zuurman L, Roy C, Schoemaker RC, Hazekamp A, den Hartigh J, Bender JC, Verpoorte R, Pinquier JL, Cohen AF, van Gerven JM (2008) Effect of intrapulmonary tetrahydrocannabinol administration in humans. J Psychopharmacol 22:707-716 